Check for updates

Cite this: RSC Adv., 2017, 7, 22524

\title{
Acoustic impedance-based manipulation of elastic microspheres using travelling surface acoustic waves†
}

\author{
Ghulam Destgeer, (D) Jin Ho Jung, Jinsoo Park, Husnain Ahmed, Kwangseok Park, \\ Raheel Ahmad and Hyung Jin Sung (D)*
}

We present a method for size-independent manipulation of elastic polystyrene (PS), poly(methyl methacrylate) (PMMA), and fused silica (FS) microspheres that uses travelling surface acoustic waves (TSAWs). Normally incident TSAWs originating from an interdigitated transducer (IDT) were used to separate similar-sized pairs of PS and PMMA or PS and FS elastic particles by producing distinct lateral deflections across laminar streamlines in a continuous flow microfluidic channel. Elastic particles with similar diameters but different acoustic impedances exhibit significantly different deflection characteristics when exposed to TSAW-based acoustic radiation forces (ARFs). For instance, exposing a mixture of PS and PMMA particles with similar diameters $(\sim 5 \mu \mathrm{m})$ to TSAWs with frequencies of 140 $\mathrm{MHz}$ and $185 \mathrm{MHz}$ produces larger deflections of the PS and PMMA particles, respectively. This difference arises because of the resonance of the elastic particles with the incoming acoustic waves at certain frequencies. Similar particle deflection characteristics were observed for mixtures of PS and FS particles with comparable diameters $(3 / 3,4.8 / 5,10 / 10 \mu \mathrm{m})$. This difference in deflection distances was used to experimentally characterize the non-linear behavior of the ARFs acting on particles $(3-10 \mu \mathrm{m})$ exposed to a range of TSAW frequencies (120-205 MHz). Our experimental results can be explained by plotting the acoustic radiation force factor $\left(F_{\mathrm{F}}\right)$ against the TSAW frequency $\left(f_{\text {TSAW }}\right)$ and the dimensionless Helmholtz number $(1<\kappa<4)$, which is calculated by using a theoretical model of an elastic microsphere suspended in a fluid.

Received 26th January 2017 Accepted 14th April 2017

DOI: $10.1039 / \mathrm{c} 7 \mathrm{ra01168g}$

rsc.li/rsc-advances sizes of the micro-objects. A few studies have reported the use of variations in the intrinsic properties such as the density or compressibility of the micro-objects for their manipulation instead of their sizes. ${ }^{23-25}$ Bourquin $e t$ al. ${ }^{23}$ tuned the density of the fluid with reference to the suspended RBCs to separate malaria-infected cells from healthy cells inside a sessile droplet. Nam et al. ${ }^{24}$ used SSAWs to continuously separate droplets with similar diameters but different densities with encapsulated cells. Augustsson et al. ${ }^{25}$ employed BAWs to realize the sizeinsensitive focusing of cells by tuning the fluid density gradient inside a microchannel. These studies utilized standing wave-based ARFs to accentuate the acoustic contrast between fluids and suspended micro-objects to achieve particle separation. However, only recently the ARFs produced by travelling acoustic waves have been used to manipulate micro-objects according to their mechanical properties instead of their sizes. ${ }^{26,27}$ Destgeer et al. ${ }^{26}$ demonstrated the separation of elastic polystyrene (PS) and poly(methyl methacrylate) (PMMA) particles of similar diameters but different acoustic impedances. Two intrinsic properties of the particles, i.e. the speed of longitudinal waves within the particle $\left(c_{1}\right)$ and the particle density $\left(\rho_{\mathrm{p}}\right)$, can be combined to compute their characteristic acoustic impedances. Differences in the values of such 
mechanical properties have also been used recently by $\mathrm{Ma}$ et $a .^{27}$ to comprehensively demonstrate the separation of PS and PMMA particles with similar diameters $(10 / 10 \mu \mathrm{m}$ and $15 /$ $15 \mu \mathrm{m})$ by tuning the frequency of applied TSAWs (45-70 $\mathrm{MHz}$ ). The non-linear responses of particles to TSAWs because of their resonances at certain frequencies open up the possibility of size-independent manipulation. However, these previous studies used lower frequencies and neglected higher resonance-modes of particle vibrations, and their methods were only applicable to PS and PMMA particles. Elastic particles with a different set of mechanical properties are expected to behave differently, so it is important to apply these findings to other particle materials.

In the present study, we achieved the size-independent manipulation of PS, PMMA, and fused silica (FS) elastic microparticles according to differences in their acoustic impedances and the corresponding ARFs, which vary with the dimensionless Helmholtz number $(\kappa)$. Particles with similar diameters $(3-10 \mu \mathrm{m})$ but different mechanical properties were exposed to TSAWs with a range of frequencies $(120-205 \mathrm{MHz})$ in order to characterize their motions for a wide range of: $1<\kappa<4$. Our findings not only corroborate previous results ${ }^{\mathbf{2 6 - 2 8}}$ for the separation of PS and PMMA particles but also provide new insights into the manipulation of PS and FS particles with smaller diameters and higher TSAW frequencies for higher Helmholtz numbers $(\kappa>2)$. The responses of particles to higher frequencies reveals new insights into their non-linear and nonintuitive behavior when exposed to TSAWs. It was observed that the first resonance mode of particle vibrations is critical to the size-independent manipulation of PS and PMMA particles; the later modes subsequently diminish rendering them as pseudo-resonances of PS particles. However, for FS particles, such resonance does not occur for $1<\kappa<4$. Therefore, FS particles exhibit a smoother transition around $\kappa \cong 1$ from being totally unaffected to being linearly deflected by ARFs.

\section{Working mechanism}

A sample fluid containing suspended elastic microparticles is sandwiched between two sheath-flows to focus the particles inside a PDMS microchannel attached to a piezoelectric lithium niobate (LN) substrate (see Fig. 1). An interdigitated transducer (IDT) deposited on top of the LN substrate generates TSAWs upon actuation by an AC signal. The TSAWs impart ARFs to the suspended elastic microparticles upon interaction with the fluid inside the microchannel. To characterize the motion of particles with diameter $d_{\mathrm{p}}$ suspended inside a fluid with speed of sound $c_{\mathrm{f}}$ exposed to acoustic waves with frequency $f_{\text {TSAW }}$ and wavelength $\lambda_{\mathrm{f}}$, the Helmholtz number $\kappa\left(=\pi f_{\text {TSAW }} d_{\mathrm{p}} / c_{\mathrm{f}}=\pi d_{\mathrm{p}} / \lambda_{\mathrm{f}}\right)$ is an important parameter used to standardize discussions of particle manipulation. The individual particles in the particle pairs (PS and PMMA, and PS and FS) deflect differently because of their non-linear responses to acoustic waves beyond $\kappa>1$, which opens up the possibility of the size-independent, acoustic impedance-based, flip-flop sorting of particles, as illustrated in Fig. 1A(i)-(iv). It has been observed that a transition in particles' response occurs at $\kappa \cong 1$ for most particle materials suspended in water. ${ }^{3,29,30}$ However, the actual value can vary slightly with changes in fluid composition and particle materials.

For $\kappa<1$, the ARF acting on a particle due to a standing wave scales with $d_{\mathrm{p}}{ }^{3}$ whereas it scales with $d_{\mathrm{p}}{ }^{6}$ for a travelling wave. ${ }^{13,17}$ However, the ARF due to travelling waves is insignificant, so the ASF-based drag force dominates the motion of the particles, and no observable deflection of the particles arises. When the frequency of the traveling wave or the particle diameter is increased such that $d_{\mathrm{p}} \gg \lambda_{\mathrm{f}}$ or $\kappa>1$, an asymmetric scattering of the travelling wave from the surface of the spherical micro-object results in a high ARF. This effect means that the shape of the micro-object can also be used as a parameter for the manipulation of micro-objects with variable surface morphologies. The ARF acting on a solid microsphere suspended in an inviscid fluid and the corresponding dimensionless force factor $\left(F_{\mathrm{F}}\right)$ can be estimated by using the rigid and elastic particle-based theories of King, ${ }^{31}$ and Hasegawa and Yosioka, ${ }^{32}$ respectively. Their theoretical models are not reproduced here for the sake of brevity. Summaries of the important equations can be found elsewhere. ${ }^{27,33,34} F_{\mathrm{F}}$ is plotted against $\kappa$ for PS, PMMA, and FS particles suspended in water-glycerol mixtures in Fig. 1B. It has been shown that for a PS particle (assumed rigid) suspended in water, the $F_{\mathrm{F}}$ curve increases for $0<\kappa<2.5$, and that $F_{\mathrm{F}}$ is very stable and constant for $\kappa>2.5$; however, for elastic PS particles considerable non-linear behavior is found in the $F_{\mathrm{F}}$ curve for $\kappa>1$ (see Fig. S1 $\dagger){ }^{34}$ The peaks in the $F_{\mathrm{F}}$ curves for the PS and PMMA particles correspond to the internal vibrations of the particles and their resonances with the incoming waves. These resonant peaks correspond to subsequent modes of particle vibration and are significant in the PS and PMMA graphs but not in that of FS. This result makes sense because the FS particles are more rigid than the elastic PS and PMMA particles..$^{33}$ Moreover, the lower speed of sound in the PS and PMMA particles also makes a difference; variations in the density and speed of sound significantly alter the $F_{\mathrm{F}}$ graphs (see Fig. $\mathrm{S} 2 \dagger$ ).

Based on the $F_{\mathrm{F}}$ plots for the different particle materials, flipflop sorting and manipulation of a mixture of similar diameter PS and PMMA particles can be achieved by tuning the frequency of incident TSAWs (see Fig. 1B). For $\kappa \cong 1.29$, the PS particles are expected to experience a significantly higher ARF than the PMMA particles, whereas the reverse effect is expected for $\kappa \cong$ 1.69 as suggested by the first peaks in their $F_{F}$ curves. A similar flip-flop manipulation of particles can be demonstrated for a combination of PS and FS particles, as shown in Fig. 1A(iii) and (iv). A very weak ARF is observed for the higher modes of vibration associated with the $3^{\text {rd }}$ or $5^{\text {th }}$ peaks in the PS curves and $\kappa>2.5$, which means that these peaks are spurious. This is consistent with the fact that the model of Hasegawa and Yosioka $^{32}$ does not take into account the attenuation of acoustic waves within the particles and the viscosity of the fluid. ${ }^{27}$

\section{Experimental section}

The polydimethylsiloxane (PDMS) microchannels (Sylgard 184, Dow Corning), having a width of $200 \mu \mathrm{m}$ (Fig. 2-4) or $500 \mu \mathrm{m}$ (Fig. 5) at the deflection zone, were fabricated by using conventional soft photolithography techniques and then bonded to the 
(A)

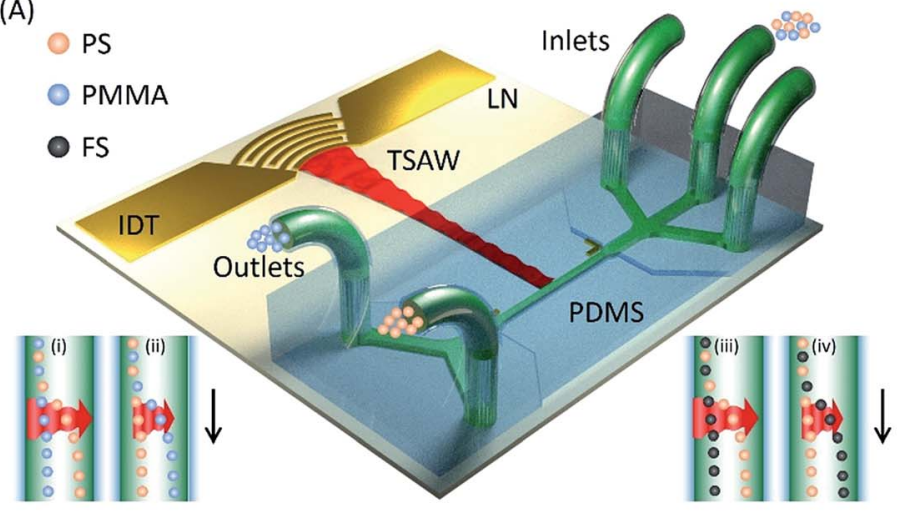

(B)

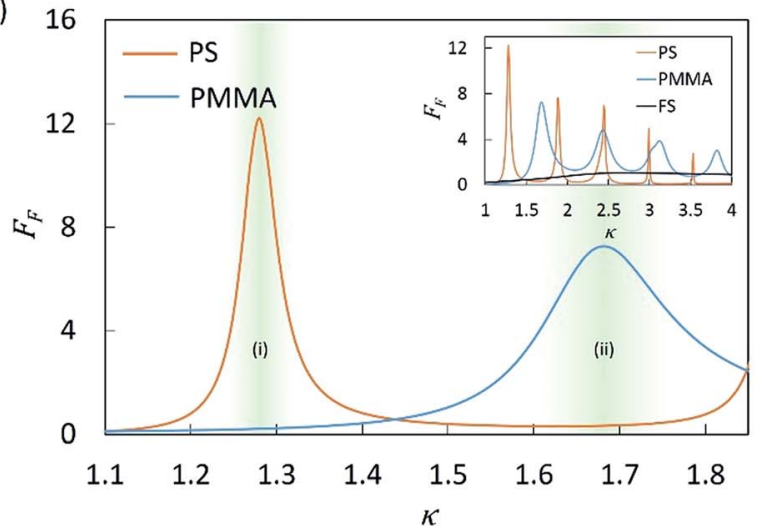

Fig. 1 (A) Schematic diagram of the acoustofluidic device composed of a polydimethylsiloxane (PDMS) microchannel bonded to a lithium niobate (LN) substrate with a focused interdigitated transducer (IDT) deposited on top. A mixture of polystyrene (PS) and poly(methyl methacrylate) (PMMA) particles or of PS and fused silica (FS) particles with similar diameters $\left(d_{p}\right)$ is pumped through the inlet port while two sheath flows focus the particles into a narrow stream. The travelling surface acoustic waves (TSAWs) originating from the IDT can deflect (i) the PS particles more than the PMMA particles or (ii) the PMMA particles more than the PS particles depending on the TSAW frequency $\left(f_{T S A W}\right)$ and the diameters of the particles. Similar behavior is expected for PS and FS particle combinations, as shown in (iii) and (iv). (B) The dimensionless acoustic radiation force factor $\left(F_{\mathrm{F}}\right)$ for PS and PMMA particles suspended in a water-glycerin mixture plotted against the dimensionless Helmholtz number $\kappa=\left(\pi d_{\mathrm{p}} f_{\mathrm{TSAW}} / c_{\mathrm{f}}\right)$. The dominance of the PS curve over the PMMA curve for $\kappa \cong 1.29$ and the dominance of the PMMA curve over the PS curve for $\kappa \cong 1.69$ correspond to the particle deflections in (i) and (ii) respectively in (A). The inset shows the corresponding plots for PS, PMMA, and FS particles for $1<\kappa<4$.

LN substrate $\left(128^{\circ} \mathrm{Y} \mathrm{X}\right.$ cut $\mathrm{LiNbO}_{3}$, MTI Korea) with oxygen plasma bonding (COVANCE-MP, Femto Science) after punching the inlet and outlet ports. ${ }^{33,35,36}$ A syringe pump (neMESYS, Cetoni $\mathrm{GmbH}$ ) was used to pump fluids through the three separate inlet ports of the microchannel. A pair of metal electrodes were deposited on top of the LN substrate by using an e-beam evaporation process to fabricate an IDT for generating the TSAWs that actuate the microparticles inside the microchannel. ${ }^{37,38}$ Focused TSAWs with resonance frequencies of approximately 129,155 , and $192 \mathrm{MHz}$ were used; however, a single device can be actuated with a range of different frequencies at the expense of a higher input power (Fig. 2, 3, and 5). ${ }^{33}$ Fig. 4 was obtained by using slanted finger IDTs actuated over a range of frequencies; the sample fluid used was water. ${ }^{39}$ A RF signal generator (N5171B, Agilent Technologies) connected to a power amplifier (LZY-22+, Mini Circuits) was used to run the acoustofluidic device mounted on the stage of a fluorescence microscope (BX53, Olympus). Experimental images were captured by using a camera (DP72, Olympus) attached to the microscope, and the images were post-processed with ImageJ software.

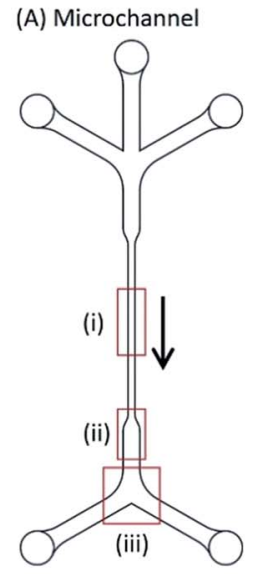

(B)

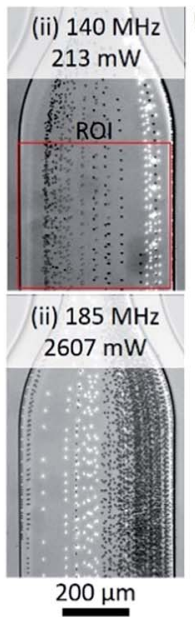

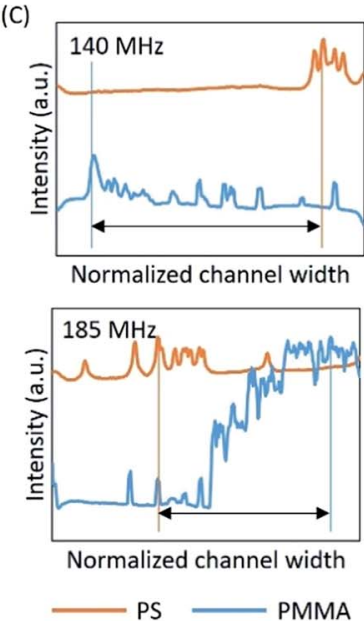

(D)

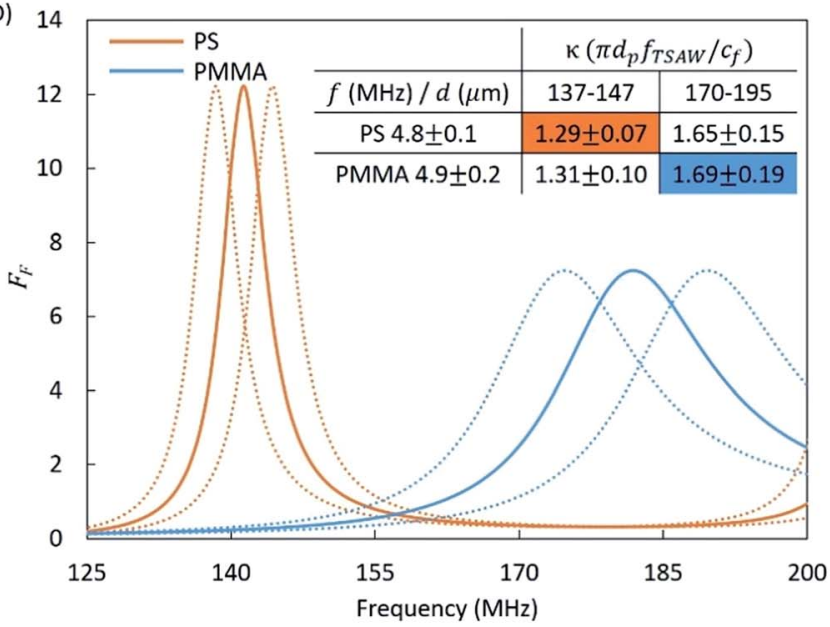

Fig. 2 (A) Schematic diagram of the microchannel; the central (i), downstream (ii), and outlet (iii) zones are shown as red rectangles. (B) Experimental images of the downstream zone after exposure of the particles to 140 and $185 \mathrm{MHz}$ TSAWs, for which maximum deflections were observed for the PS (white) and PMMA (black) particles, respectively. (C) Intensity profiles with respect to the normalized channel width indicating the locations of the particles. (D) The acoustic radiation force factor $\left(F_{\mathrm{F}}\right)$ is plotted against the frequency of the acoustic wave; the maxima are present in the PS $4.8 \mu \mathrm{m}$ particle curve near $140 \mathrm{MHz}$ and near $180 \mathrm{MHz}$ in the PMMA $4.9 \mu \mathrm{m}$ particle curve, in good agreement with the experimental results. The dotted curves are those for the standard deviations in the particle diameters. 
(A) Central actuation zone (i)

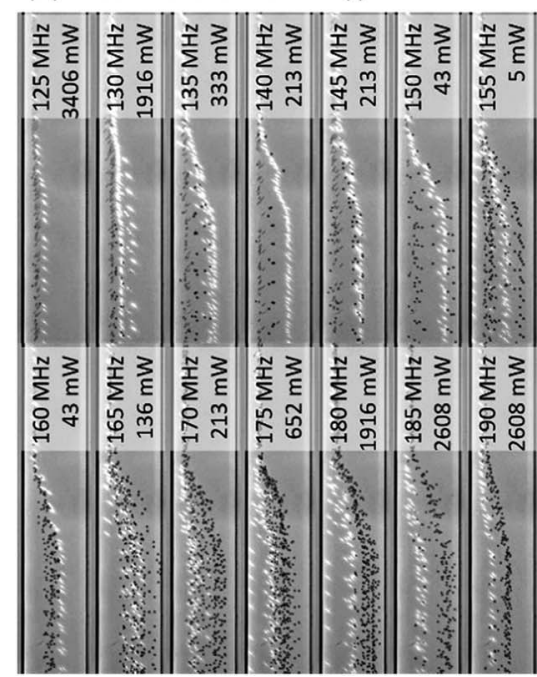

(B) Outlet zone (iii)

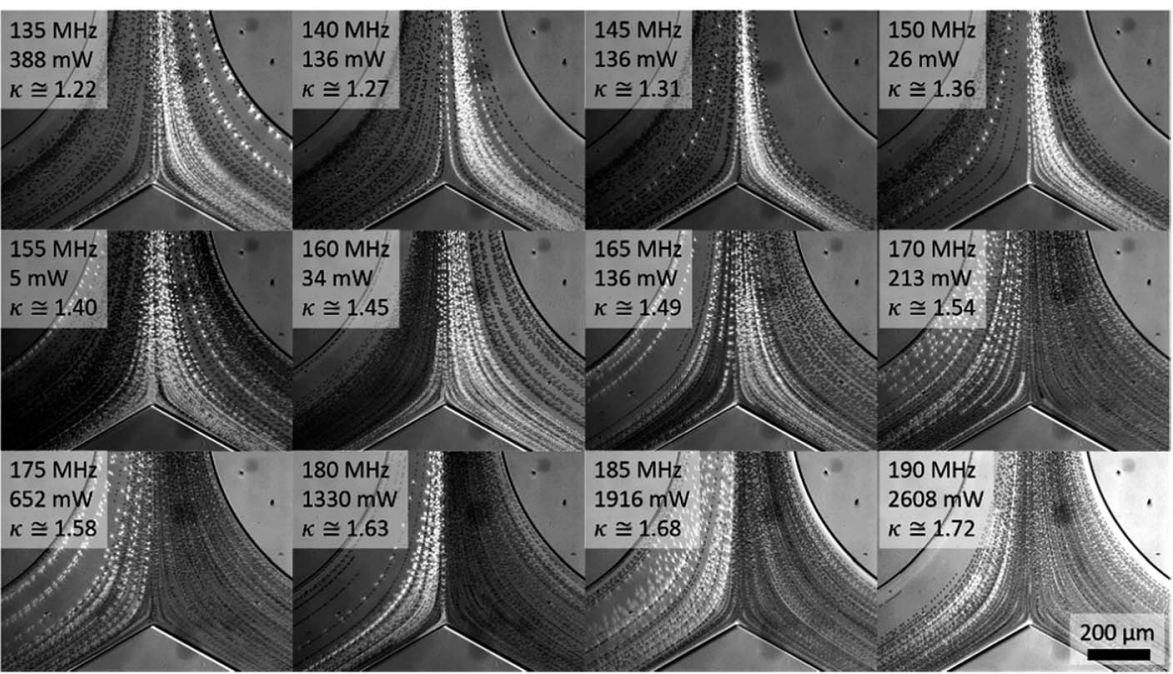

Fig. 3 (A) Images of the central actuation zone of the microchannel when a mixture of PS ( $4.8 \mu \mathrm{m}$, white) and PMMA (4.9 $\mu \mathrm{m}$, black) particles is exposed to a range of different SAW frequencies and input powers. The deflection of the PS particles is greater than that of the PMMA particles for frequencies in the range 125-150 MHz. For frequencies in the range 155-165 MHz, the deflections of the two types of particles are quite similar and it is difficult to distinguish their positions. For frequencies in the range 170-190 MHz, the PMMA particles are deflected more than the PS particles. (B) Images of the outlet zone as the particles exit through different outlet ports after exposure to frequencies in the range $135-190 \mathrm{MHz}$. The outlet flow rates are balanced, both at $100 \mu \mathrm{L} \mathrm{h} \mathrm{h}^{-1}$. For frequencies in the range $135-145 \mathrm{MHz}$, most of the fluorescent PS particles exit through the right outlet while the PMMA particles exit through the left outlet. When the frequency is in the range $150-165 \mathrm{MHz}$, the distinction is unclear. For frequencies in the range 170-190 MHz, the PMMA particles prefer the right outlet and the PS particles prefer the left outlet.

$\begin{array}{lllllllllllll}\text { (A) } 135 \mathrm{MHz} & 145 \mathrm{MHz} & 150 \mathrm{MHz} & 165 \mathrm{MHz} & 185 \mathrm{MHz} & \text { (B) } 165 \mathrm{MHz} & 170 \mathrm{MHz} & 175 \mathrm{MHz} & 180 \mathrm{MHz} & 185 \mathrm{MHz} & \text { (C) }\end{array}$
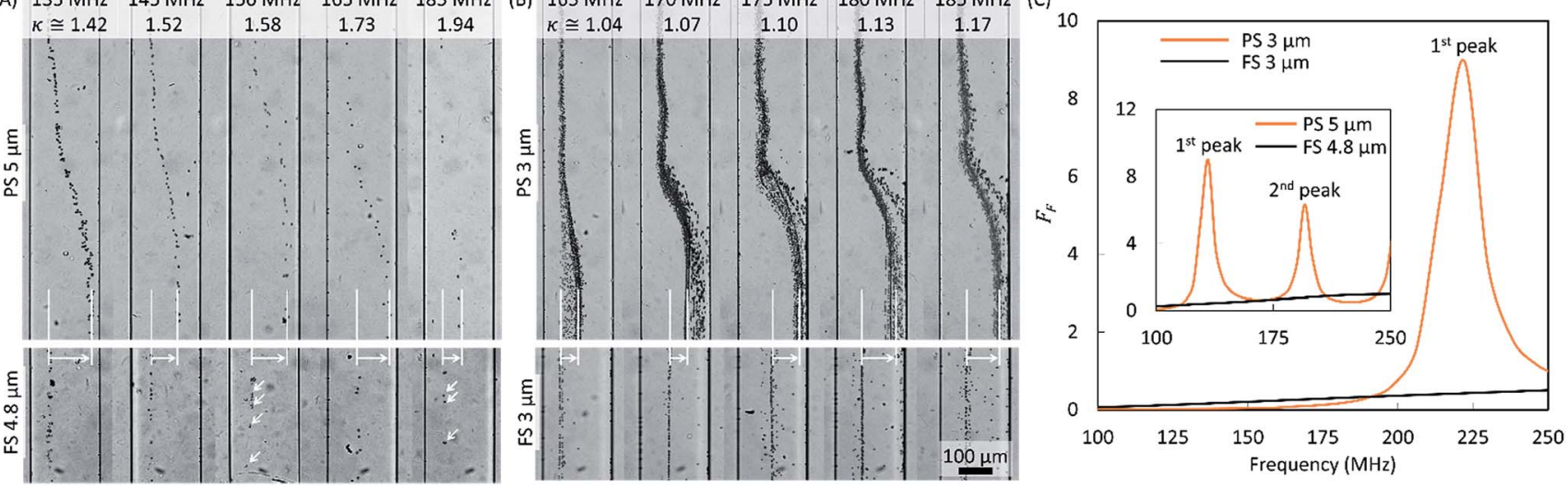

Fig. 4 (A) $5 \mu \mathrm{m}$ PS particles deflect more than $4.8 \mu \mathrm{m}$ FS particles when exposed to TSAWs with frequencies in the range $135-185 \mathrm{MHz}$, which corresponds to the $1^{\text {st }}$ and $2^{\text {nd }}$ peaks in the curve for PS particles in (C). (B) Similarly, $3 \mu \mathrm{m}$ PS particles deflect more than $3 \mu \mathrm{m}$ FS particles when exposed to TSAWs with frequencies in the range $165-185 \mathrm{MHz}$. (C) The variations in the force factor with frequency for particles of PS and FS with different diameters.

The particle mixtures were prepared in a $40 \mathrm{w} / \mathrm{w} \%$ glycerol solution in water (unless otherwise mentioned) to obtain a fluid density of $1.09 \mathrm{~g} \mathrm{~cm}^{-3}$, which is higher than that of the PS particles, $1.05 \mathrm{~g} \mathrm{~cm}^{-3}$, and lower than those of the PMMA particles, $1.18 \mathrm{~g} \mathrm{~cm}^{-3}$, and the FS particles, $2.1 \mathrm{~g} \mathrm{~cm}^{-3}$. The effects of varying the density on the force factor are significant according to the theoretical model of Hasegawa and Yosioka ${ }^{32}$ (see Fig. S2 †). PS particles (Thermo Scientific) with diameters of $3 \mu \mathrm{m}$ (NIST; $3.005 \pm 0.027 \mu \mathrm{m}$ ), $4.8 \mu \mathrm{m}$ (green fluorescent; uniformity < 5\%), $5 \mu \mathrm{m}$ (NIST; $5.021 \pm 0.041 \mu \mathrm{m}$ ), and $10 \mu \mathrm{m}$ (green fluorescent; uniformity $<5 \%$ ), PMMA particles
(Magsphere Inc.) with a diameter of $4.9 \mu \mathrm{m}( \pm 0.2 \mu \mathrm{m})$, and FS particles with diameters of $3 \mu \mathrm{m}( \pm 0.15 \mu \mathrm{m}$; Polysciences Inc.), $4.8 \mu \mathrm{m}( \pm 0.3$; Bangs Laboratories, Inc.), and $10 \mu \mathrm{m}$ (plain green fluorescence, Microspheres-Nanospheres Inc.) were used. To prepare the particle samples for the experiments, $1 \%$ Tween 20 (Tokyo Chemical Industry Co., Ltd.) was added to $1 \mathrm{~mL}$ aqueous-glycerol solutions containing $50 \mu \mathrm{L}$ of PS and PMMA particle solution or PS and FS particle solution, unless otherwise mentioned. The characteristic acoustic impedances of the particles were calculated with the formula $Z_{\mathrm{p}}=\rho_{\mathrm{p}} c_{1}$ and are listed in Table 1. The FS particles clearly have a much higher $Z_{\mathrm{p}}$ 


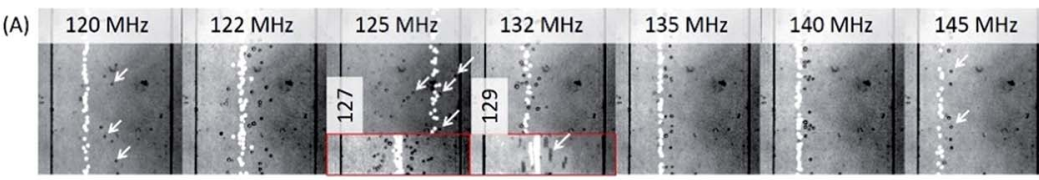

(B) $185 \mathrm{MHz} \quad 187 \mathrm{MHz}$

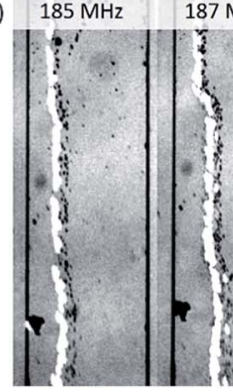

$190 \mathrm{MHz}$

$192 \mathrm{MHz}$

$195 \mathrm{MHz}$

$200 \mathrm{MHz}$
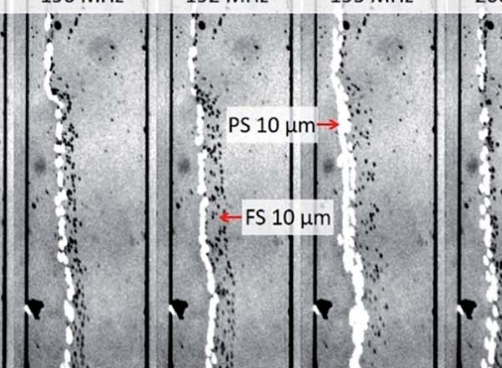

$205 \mathrm{MHz}$

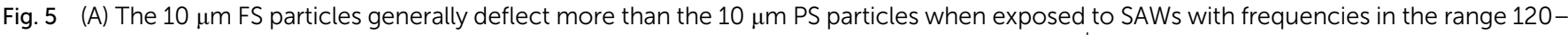

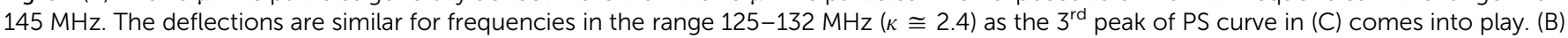

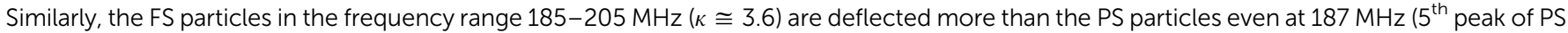

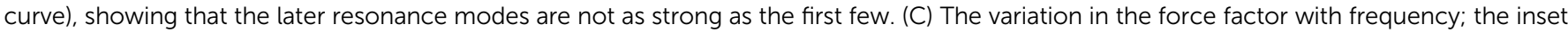
shows the force factor curves for $2<\kappa<4$.

Table 1 Material properties

\begin{tabular}{|c|c|c|c|c|c|}
\hline Properties & PS & PMMA & FS & Water & Aqua-glycerol \\
\hline Density $\rho_{\mathrm{p}}, \rho_{\mathrm{f}}\left(\mathrm{g} \mathrm{cm}^{-3}\right)$ & 1.05 & 1.18 & 2.21 & 0.998 & 1.09 \\
\hline $\begin{array}{l}\text { Speed of sound } c_{\mathrm{l}}, c_{\mathrm{f}} \\
\text { (longitudinal) }\left(\mathrm{m} \mathrm{s}^{-1}\right)\end{array}$ & 2350 & 2757 & 5950 & 1495 & 1665 \\
\hline $\begin{array}{l}\text { Speed of sound } c_{\mathrm{s}} \\
\text { (shear) }\left(\mathrm{m} \mathrm{s}^{-1}\right)\end{array}$ & 1120 & 1400 & 3750 & - & - \\
\hline Poisson's ratio $\sigma$ & 0.35 & 0.35 & 0.17 & - & - \\
\hline $\begin{array}{l}\text { Acoustic impedance } \\
Z_{\mathrm{p}}, Z_{\mathrm{f}} \text { (MRayls) }\end{array}$ & 2.47 & 3.25 & 13.15 & 1.49 & 1.81 \\
\hline
\end{tabular}

than the PS and PMMA particles. A difference in the $Z_{\mathrm{p}}$ values can be used to enable the size-independent manipulation of particles with different densities and speeds of sound.

\section{Results and discussion}

A mixture of PS (fluorescent green) and PMMA particles with average diameters of 4.8 and $4.9 \mu \mathrm{m}$ was pumped through a microchannel and exposed to TSAWs: the PS particles are deflected more than the PMMA particles at a frequency of 140 $\mathrm{MHz}$ and at $185 \mathrm{MHz}$ the converse arises (see Fig. 2A and B). A net flow rate of $200 \mu \mathrm{L} \mathrm{h}^{-1}$ was used to focus the sample fluid carrying the particles $\left(5 \mu \mathrm{L} \mathrm{h}^{-1}\right)$ by applying sheath flows (left $20 \mu \mathrm{L} \mathrm{h}^{-1}$ and right $175 \mu \mathrm{L} \mathrm{h}^{-1}$ ) to align the particles close to the left wall of the microchannel to allow for a wider deflection distance and to avoid the microchannel anechoic corner. ${ }^{39}$ The deflections of the PS particles and PMMA particles are compared by plotting the intensity profiles against the normalized channel widths for the downstream zone (ii) in Fig. 2C. The experimental results can be explained by examining the plots of the force factor against the acoustic wave frequency: the first maxima in the PS and PMMA curves are observed at 140 and $180 \mathrm{MHz}$ respectively (see Fig. 2D). Images of the central (i) and outlet (iii) zones of the microchannel are shown in Fig. 3A and B for frequencies in the range 125-190
MHz. The outward flow rates at the two outlet ports in Fig. 3B were fixed at $100 \mu \mathrm{L} \mathrm{h}^{-1}$. The flip-flop manipulation of particles was confirmed: the greater deflection of PS particles when compared to that of PMMA particles for the frequency range $125-150 \mathrm{MHz}$ is reversed for frequencies greater than $170 \mathrm{MHz}$ (see Fig. 3A). These results are in good agreement with the force factor plots in Fig. 2D, which show a transition in the particles' deflection behaviors in the frequency range 150-165 MHz. The flows of particles through the separate outlet ports exhibit similar behavior, as shown in Fig. 3B: the PS and PMMA particles switch ports when the frequency is increased. The wider distribution in the PMMA particle deflected locations arises because their standard deviation $( \pm 0.2 \mu \mathrm{m})$ is greater than that of the PS particles $( \pm 0.1 \mu \mathrm{m})$. Moreover, see Fig. S3 and S4 in the ESI $\dagger$ for additional results.

Fig. 4 demonstrates the manipulation of PS ( 3 and $5 \mu \mathrm{m}$ ) and FS ( 3 and $4.8 \mu \mathrm{m}$ ) particles for a wide range of TSAW frequencies (135-185 MHz). The PS particles are clearly deflected more than the FS particles at all frequencies (see Fig. $4 \mathrm{~A}$ and $\mathrm{B}$ ). The variation in the force factor with frequency in Fig. $4 \mathrm{C}$ shows the $1^{\text {st }}$ and $2^{\text {nd }}$ maxima for the $5 \mu \mathrm{m}$ PS curve, which clearly dominates the FS curve. However, PS particles with a diameter of $3 \mu \mathrm{m}$ are deflected more than $3 \mu \mathrm{m}$ FS particles even though the $F_{\mathrm{F}}$ curve for the FS particles lies slightly above the PS curve (165-185 MHz). One possible explanation is that the PS particles experience a strong ARF due to the strong first vibration mode of the particles corresponding to the $1^{\text {st }}$ peak in the curve. The ARF starts to dominate the motion of the PS particles beyond $\kappa \cong 1$ and reaches a maximum at $\kappa \cong 1.3$, whereas the ARF acting on the FS particles reaches its maximum at $\kappa \cong 2.5$. This phenomenon is reversed in Fig. 5: the $10 \mu \mathrm{m}$ FS particles are deflected more than the $10 \mu \mathrm{m}$ PS particles because of the domination of the FS curve for $\kappa>2$. For TSAW frequencies in the range 120-145 MHz (see Fig. 5A), the FS particles are generally deflected more; however, for the frequency range 125-132 MHz, the deflection of the FS particles is similar to that of the PS particles as the $3^{\text {rd }}$ peak in the PS curve near $\kappa \cong 2.4$ 
comes into play. It is clear that the strength of this mode is not comparable to those of the first two modes of particle vibration. The deflection of the FS particles exceeds that of the PS particles for frequencies in the range 185-205 MHz (see Fig. 5B). The $5^{\text {th }}$ mode of vibration of the PS particles, at $187 \mathrm{MHz}$ and $\kappa \cong 3.6$ (see Fig. 5C), does not have a strong influence because the FS particles are clearly deflected more than the PS particles. It is evident from these experimental observations that the $1^{\text {st }}$ mode of vibration of the elastic PS particles is the strongest, and that the strength of the higher modes decreases gradually. As pointed out by Ma et $a l .{ }^{27}$ the discrepancies between the experimental results and theoretical predictions arise because the theoretical model does not consider wave absorption within the particles because of the unavailability of the attenuation coefficients within the particles. $^{\mathbf{4 0 , 4 1}}$ Moreover, the viscous nature of the carrier fluid has not been considered in the theoretical models that could also affect the force factor curves to some extent. This experimental investigation could be extended to new particle materials and the theoretical model could be improved in future by the incorporation of the attenuation coefficients and the modeling of wave propagation within the particles.

\section{Conclusions}

The differences between the acoustic impedances of elastic microspheres have been used to manipulate the combinations of similar-sized PS, PMMA, and FS particles by using TSAWs. Elastic microspheres exhibit non-linear behavior when high frequency TSAWs interact with them, which induces a resonance in the particles that corresponds to a spike in the force factor curve. These spikes occur at different positions (or $\kappa$ values), which enables the selective deflection of the particle of choice by tuning the frequency of the incident acoustic wave or the particle diameter. A higher regime of $\kappa$ values was explored in this study to investigate the effects of the higher resonant modes of particles on deflection distances. The first two peaks in the force factor curve give rise to the strongest ARFs on the PS particles, and the higher peaks do not give rise to significant deflections of particles. The variation in the forces acting on the particles at different frequencies results in a flip-flop sorting mechanism in which one particle is deflected more than the other, and vice versa.

\section{Authors contributions}

G. D. conceived the research. H. J. S. supervised the research. G. D., J. H. J., J. P., and K. P. designed the experiments. G. D., H. A., and R. A. performed the experiments. G. D., J. H. J., J. P., H. A., K. P., R. A., and H. J. S. contributed to the manuscript preparation. All authors have given approval to the final version of the manuscript.

\section{Acknowledgements}

This work was supported by the KUSTAR-KAIST Institute, and the Creative Research Initiatives (No. 2017-013369) program of the National Research Foundation of Korea.

\section{Notes and references}

1 A. A. S. Bhagat, H. Bow, H. W. Hou, S. J. Tan, J. Han and

C. T. Lim, Med. Biol. Eng. Comput., 2010, 48, 999-1014.

2 N. Pamme, Lab Chip, 2007, 7, 1644-1659.

3 G. Destgeer and H. J. Sung, Lab Chip, 2015, 15, 2722-2738.

4 G. Destgeer, H. Cho, B. H. Ha, J. H. Jung, J. Park and H. J. Sung, Lab Chip, 2016, 16, 660-667.

5 L. Y. Yeo and J. R. Friend, Annu. Rev. Fluid Mech., 2014, 46, 379-406.

6 X. Ding, P. Li, S.-C. S. Lin, Z. S. Stratton, N. Nama, F. Guo, D. Slotcavage, X. Mao, J. Shi, F. Costanzo and T. J. Huang, Lab Chip, 2013, 13, 3626-3649.

7 A. Fornell, J. Nilsson, L. Jonsson, P. K. Periyannan Rajeswari, H. N. Joensson and M. Tenje, Anal. Chem., 2015, 87, 1052110526.

8 I. Leibacher, P. Reichert and J. Dual, Lab Chip, 2015, 15, 2896-2905.

9 O. Jakobsson, S. S. Oh, M. Antfolk, M. Eisenstein, T. Laurell and H. T. Soh, Anal. Chem., 2015, 87, 8497-8502.

10 M. Antfolk, C. Magnusson, P. Augustsson, H. Lilja and T. Laurell, Anal. Chem., 2015, 87, 9322-9328.

11 A. R. Rezk, L. Y. Yeo and J. R. Friend, Langmuir, 2014, 30, 11243-11247.

12 G. Destgeer, B. Ha, J. Park and H. J. Sung, Anal. Chem., 2016, 88, 3976-3981.

13 G. Destgeer, K. H. Lee, J. H. Jung, A. Alazzam and H. J. Sung, Lab Chip, 2013, 13, 4210-4216.

14 D. J. Collins, A. Neild and Y. Ai, Lab Chip, 2015, 16, 471-479. 15 J. H. Jung, G. Destgeer, B. Ha, J. Park and H. J. Sung, Lab Chip, 2016, 16, 3235-3243.

16 G. Destgeer, J. H. Jung, J. Park, H. Ahmed and H. J. Sung, Anal. Chem., 2017, 89, 736-744.

17 D. J. Collins, Z. Ma, J. Han and Y. Ai, Lab Chip, 2017, 112, 4469-4506.

18 Z. Ma, A. Teo, S. Tan, Y. Ai and N.-T. Nguyen, Micromachines, 2016, 7, 216.

19 J. Shi, H. Huang, Z. Stratton, Y. Huang and T. J. Huang, Lab Chip, 2009, 9, 3354-3359.

20 P. Li, Z. Mao, Z. Peng, L. Zhou, Y. Chen, P. Huang, C. I. Truica, J. J. Drabick, W. S. El-Deiry, M. Dao, S. Suresh and T. J. Huang, Proc. Natl. Acad. Sci. U. S. A., 2015, 112, 4970-4975.

21 Y. Ai, C. K. Sanders and B. L. Marrone, Anal. Chem., 2013, 85, 9126-9134.

22 J. Nam, H. Lim, D. Kim and S. Shin, Lab Chip, 2011, 11, 3361. 23 Y. Bourquin, A. Syed, J. Reboud, L. C. Ranford-Cartwright, M. P. Barrett and J. M. Cooper, Angew. Chem., 2014, 126, 5693-5696.

24 J. Nam, H. Lim, C. Kim, J. Yoon Kang and S. Shin, Biomicrofluidics, 2012, 6, 24120.

25 P. Augustsson, J. T. Karlsen, H.-W. Su, H. Bruus and J. Voldman, Nat. Commun., 2016, 7, 11556.

26 G. Destgeer, B. H. B. H. Ha, J. S. J. S. Park, J. H. J. H. Jung, A. Alazzam and H. J. H. J. Sung, in 19th International Conference on Miniaturized Systems for Chemistry and Life 
Sciences October 25-29, 2015, Gyeongju, Korea, 2015, pp. 1014-1016.

27 Z. Ma, D. J. Collins, J. Guo and Y. Ai, Anal. Chem., 2016, 88, 11844-11851.

28 H. Ahmed, G. Destgeer, J. Park, J. H. Jung, and H. J. Sung, in Acoustofluidics 2016, September 22-23, DTU, Denmark, 2016, pp. 73-74.

29 V. Skowronek, R. W. Rambach, L. Schmid, K. Haase and T. Franke, Anal. Chem., 2013, 85, 9955-9959.

30 V. Skowronek, R. W. Rambach and T. Franke, Microfluid. Nanofluid., 2015, 19, 335-341.

31 L. V. King, Proc. R. Soc. A, 1934, 147, 212-240.

32 T. Hasegawa and K. Yosioka, J. Acoust. Soc. Am., 1969, 46, 1139.

33 G. Destgeer, B. H. Ha, J. H. Jung and H. J. Sung, Lab Chip, 2014, 14, 4665-4672.
34 G. Destgeer, B. H. B. H. Ha, J. Park, J. H. J. H. Jung, A. Alazzam and H. J. Sung, Phys. Procedia, 2015, 70, 34-37.

35 B. Ha, J. Park, G. Destgeer, J. H. J. H. Jung and H. J. Sung, Anal. Chem., 2016, 88, 4205-4210.

36 H. W. Kang, H. J. Sung, T.-M. Lee, D.-S. Kim and C.-J. Kim, J. Micromech. Microeng., 2009, 19, 15025.

37 G. Destgeer, A. Alazzam and H. J. H. J. Sung, J. Mech. Sci. Technol., 2016, 30, 3945-3952.

38 G. Destgeer, S. Im, B. H. Ha, J. H. Jung, M. A. Ansari and H. J. Sung, Appl. Phys. Lett., 2014, 104, 23506.

39 G. Destgeer, B. H. Ha, J. Park, J. H. Jung, A. Alazzam and H. J. Sung, Anal. Chem., 2015, 87, 4627-4632.

40 T. Hasegawa and Y. Watanabe, J. Acoust. Soc. Am., 1978, 63, 1733.

41 F. G. Mitri, Wave Motion, 2005, 43, 12-19. 